\title{
WIE KOMMT DER KLIMAWANDEL ZU DEN HISTORISCHEN GÄRTEN?
}

\begin{abstract}
In unserem Beitrag fragen wir danach, inwieweit der Klimawandel zu einem expliziten Thema für die historischen Gärten in Berlin-Brandenburg bzw. Ostdeutschland geworden ist. Für die Bearbeitung dieser aktuellen Fragestellung rekonstruieren wir bisherige, vom Thema Klimawandel unabhängige gesellschaftliche Bedeutungszuschreibungen historischer Gärten im Zeitverlauf von 1918 bis heute. Zuspitzend arbeiten wir die idealtypischen Gegensätze zwischen der Zuschreibung als historischer Garten einerseits oder als Volkspark andererseits heraus und skizzieren ausschnitthaft die damit verbundenen Debatten. Der Beitrag experimentiert mit einem eher ungewöhnlichen Format eines kommentierten Interviews und will die Leserinnen und Leser zur Teilhabe am Zwiegespräch der Autorin und des Autors einladen. Originalzitate von Hartmut Dorgerloh werden mit einem soziologischen Interpretationsangebot institutionellen Wandels gerahmt.
\end{abstract}

In our article we look at the extent to which climate change has become an explicit topic for historic gardens in Berlin-Brandenburg and eastern Germany. To address this topical issue, we reconstruct earlier societal interpretations of gardens between 1918 and today that are independent of the subject of climate change. Taking this one step further, we determine the ideal contrasts between classification as historic gardens on the one hand and people's parks on the other, and touch briefly on the related debates. This article experiments with the somewhat unusual format of a commented interview and wishes to invite the readers to participate in this dialogue between the two authors. Original quotes from Hartmut Dorgerloh are framed with a sociological interpretation of institutional change.

Im Text werden Maskulinum und Femininum verwendet, wenn es um Personen geht. Gemeint sind grundsätzlich alle Menschen, gleich welcher Geschlechtsidentität sie sich zugehörig fühlen. 


\section{Einleitung}

Der anthropogene Klimawandel geistert einem Gespenst gleich seit einigen Jahren durch die Weltgeschichte. Er erscheint bedrohlich, ist schwer dingfest zu machen, hat unscharfe Konturen, wird in seiner Existenz angezweifelt und treibt ein vielfältiges, wirkmächtiges, aber nicht immer klar zuordenbares Unwesen. Die Diskussionen um den und der Umgang mit dem Klimawandel sind eine Herausforderung, auch für die historischen Gärten. In unserem Beitrag fragen wir danach, inwieweit der Klimawandel zu einem expliziten Thema für die historischen Gärten in Berlin-Brandenburg bzw. Ostdeutschland geworden ist oder werden könnte.

Für die Bearbeitung dieser aktuellen Fragestellung bietet es sich an, bisherige Rahmungen historischer Gärten zu rekonstruieren. Wir betten unsere Frage deshalb in einen weiteren Kontext ein und erkunden vom Thema Klimawandel unabhängige gesellschaftliche Bedeutungszuschreibungen von historischen Gärten im Zeitverlauf. Dazu wagen wir einen skizzenhaften Rückblick auf die Geschichte der historischen Gärten, beginnend mit dem Ende der Monarchie und mit besonderem Fokus auf den Stellenwert der Gärten in der DDR und die wechselvollen Zeiten der Wende bis hin zu den gegenwärtigen Entwicklungen. Dieser Rekonstruktion gesellen wir einen soziologischen Erklärungsversuch von Kontinuitäten und Diskontinuitäten, von institutioneller Beharrlichkeit und institutionellem Wandel hinzu. Am Beispiel verschiedener Kulminationspunkte erscheinen uns diese Dynamiken besonders deutlich sichtbar. Wir wenden uns deshalb bestimmten Phasen und besonderen Gegebenheiten etwas ausführlicher zu: a) dem Ende der Monarchie und dem Beginn der Erhaltung historischer Gärten, b) den Kontinuitäten der gärtnerischen Fachpflege, c) der DDR und der Gartendenkmalpflege, d) der Wende, dem Park Babelsberg und der Mauer, e) der Nachwendezeit und der Debatte um den Parkeintritt in Sanssouci und f) dem Thema Klimawandel und den historischen Gärten in Berlin und Potsdam.

Der Beitrag experimentiert mit einem eher ungewöhnlichen Format, das dem interdisziplinären Charakter der Arbeitsgruppe »Historische Gärten im Klimawandel « der BerlinBrandenburgischen Akademie der Wissenschaften (BBAW) Rechnung trägt. Wir wollen die Leserinnen und Leser am Zwiegespräch des Autors und der Autorin teilhaben lassen. Hartmut Dorgerloh war von 2001 bis 2018 Generaldirektor der Stiftung Preußische Schlösser und Gärten Berlin-Brandenburg (SPSG) und ist als Kunsthistoriker und Kulturmanager wie kaum ein anderer mit der Berlin-Potsdamer Kulturlandschaft vertraut. Stefanie Hiß ist als Sozialwissenschaftlerin seit nunmehr 15 Jahren mit dem Thema institutioneller Wandel und Nachhaltigkeit befasst. Den entstandenen Austausch fassen wir in ein Format, das wohl am besten als kommentiertes Interview bezeichnet werden könnte. Ausgewählte und durchaus längere Sequenzen lassen Hartmut Dorgerloh im Original aus unserem Gesprächsmitschnitt zu Wort kommen. ${ }^{1}$ Diese Zitationen werden gerahmt durch die 
soziologische Perspektive institutionellen Wandels, die von beiden Seiten im Austausch als treffende Interpretation des Geschehens empfunden wurde. Mit diesem Format geht allerdings auch einher, dass wir im Sinne eines explorativen Verfahrens lediglich Anregungen für weitere Forschung geben. Zudem hinterlegen wir die Originalzitation nicht mit einem Literaturapparat, geben aber Hinweise zu weiterführender Lektüre. Der vorliegende Artikel ist damit kein im strengen Sinne wissenschaftlicher Beitrag, sondern ein Angebot zur Teilhabe am Zwiegespräch.

\section{Soziologische Vorbemerkungen}

Für eine Annäherung an die Frage nach der Bedeutungszuschreibung historischer Gärten im Zeitverlauf ziehen wir die Perspektive institutionellen Wandels heran, wie sie im neuen soziologischen Institutionalismus (Greenwood et al. 2017) verhandelt wird. Institutionen werden dort als Ergebnis sozialer Konstruktionen verstanden. Sie sind demnach gemeinsam geteilte und für selbstverständlich erachtete Strukturen, Praktiken und Bedeutungssysteme, die durch ihre wiederholte Anwendung auf Dauer gestellt sind und das Handeln der Individuen sowohl anleiten als auch begrenzen. Wir konzentrieren uns in diesem Beitrag vor allem auf die Bedeutung (»meaning«) als konstituierenden Aspekt von Institutionen, der das Ideelle und Symbolische betont (Zilber 2017, 419).

Inwieweit den ehemaligen höfischen Gärten als Institution in unserer Gesellschaft die Bedeutung eines schützenswerten Denkmals zugeschrieben wird, ist im Zeitverlauf immer wieder neu verhandelt worden. Die Erfüllung der mit der jeweiligen Bedeutungszuschreibung einhergehenden gesellschaftlichen Erwartungen ist für den Weiterbestand relevant, liefert sie doch die für die Institution notwendige Legitimation. Dem liegt die Annahme des neuen soziologischen Institutionalismus zu Grunde, dass die Legitimation von Institutionen durch die Akteure (auch Stakeholder) im gleichen Themenfeld (»issue-field «, Hoffman 1999) zugesprochen werden muss, damit die entscheidenden Ressourcen materieller und immaterieller Art für die Sicherung des Fortbestands fließen können. Ein historischer Garten erhält etwa finanzielle Zuweisungen oder ist durch die Respektierung seiner Parkordnung geschützt, wenn die Bedeutungszuschreibung als Gartendenkmal auch im Feld geteilt wird.

Für die ehemaligen höfischen Gärten erkennen wir bei der nachfolgenden Rekonstruktion im Zeitverlauf zuvorderst das Ringen um die vorherrschende Bedeutung, welche Rolle sie in der Gesellschaft einnehmen sollen. Zuspitzend arbeiten wir den Gegensatz zwischen zwei idealtypischen Bedeutungszuschreibungen heraus: als historischer Garten einerseits und als Volkspark andererseits. Die erste Lesart versteht den historischen Garten als einen in der Regel denkmalgeschützten Ort des Bewahrens einer vergangenen Epoche, wohingegen die zweite Lesart einen Volksgarten mit einem Ort der Freizeitgestaltung assoziiert.

Welche Lesart die Oberhand gewinnt, ist abhängig von den Akteuren, die sich darum bemühen. Die Akteure werden tätig, ringen mehr oder weniger konflikthaft um die vorherrschende Deutung und arbeiten dafür an der Bedeutungszuschreibung der Institution 
(»institutional work«). Lawrence und Suddaby $(2006,215)$ entwickelten das Konzept institutioneller Arbeit als »the purposive action of individuals and organizations aimed at creating, maintaining, and disrupting institutions «. Dem liegt die konzeptionelle Idee zu Grunde, dass Akteure mit verschiedenen Mitteln versuchen, ihre Sicht, ihre Deutung, ihre Bedeutungszuschreibung zu erschaffen, sie zu erhalten, sie durch Zerstörung anderer Deutungen zu etablieren oder sie durch institutionellen Wandel mit einfließen zu lassen.

Mit dem Konzept institutioneller Arbeit hält die Vorstellung von aktiven und zur Reflexion fähigen Akteuren Einzug in die neo-institutionalistische Theorie. Die Akteure folgen den sie umgebenden Institutionen nicht blind ("passive actor», DiMaggio 1988), sondern nehmen sie bewusst zur Kenntnis und setzen sich gestaltend damit auseinander. Bezogen auf die Gärten erlaubt uns die soziologische Perspektive institutioneller Arbeit, den Blick auf das kontinuierliche Ringen um die Bedeutungszuschreibung des historischen Gartens als Institution zu schärfen.

Auf zwei Mittel für diese fortlaufende Arbeit an der Bedeutung von Institutionen gehen wir besonders ein, nämlich den Diskurs und die Theoretisierung. Maguire und Hardy (2009) haben eindrucksvoll am Beispiel des Insektizids DDT gezeigt, wie eine ursprünglich als großartige Neuerung gefeierte Chemikalie nach einer Diskursverschiebung wenige Jahre später als hochgefährliches Gift von den Äckern wieder verbannt wurde. Munir und Phillips (2005) haben in ihrer Analyse des Kodak-Moments die Metamorphose des Fotografierens nachgezeichnet. Dem Unternehmen Kodak war es durch Diskursbeeinflussung gelungen, eine Akzeptanz für die Verwendung der neuen Technologie des Rollenfilms herzustellen und damit die Praxis des Fotografierens grundlegend zu ändern. Im Ergebnis war das Fotografieren nicht mehr nur ausgebildeten Fotografen vorbehalten, sondern wurde zu einer alläglichen Angelegenheit für alle.

Ein weiteres Mittel zur Arbeit an Institutionen ist das Theoretisieren (»theorizing«). Anlässlich einer Interruption (»jolt«) werden lokal neue Lösungen erprobt, die dann in einem nächsten Schritt durch Abstrahierung auch als Lösungsschemata für andere Regionen oder Gegebenheiten genutzt werden können (Greenwood et al. 2002).

Dieser kurze theoretische Abriss darf als stark vereinfachende Skizze einer weitverzweigten und unübersichtlichen soziologischen Debatte mit diversen Definitionsversuchen und konkurrierenden Ansätzen verstanden werden (Hampel et al. 2017; Zilber 2017). Im Folgenden wenden wir uns der Rekonstruktion des Bedeutungswandels historischer Gärten anhand ausgewählter Phasen zu.

\section{Phase a) \\ Das Ende der Monarchie und der Beginn der Erhaltung historischer Gärten}

Bis zur Abschaffung der Monarchie war der Garten in der Regel Teil einer der Öffentlichkeit nur bedingt zugänglichen Schlossanlage und wurde an die Bedürfnisse, Moden und Vorlieben der jeweiligen Eigentümer angepasst. Für unsere Betrachtungen ist insofern das 
Jahr 1918 ein passender Anfangspunkt, weil mit dem Ende der Monarchie die Gärten in die öffentliche Verwaltung übergingen. Der Gedanke, dass ein Garten bedeutsam ist, weil mit seiner Bewahrung eine bestimmte Gestaltepoche für zukünftige Generationen als Denkmal überdauern sollte, entstand in der damaligen Zeit ${ }^{2}$. Dem Garten eine Bedeutungszuschreibung als »historischer Garten « zu geben, verweist im Kern auf das Bewahren und nicht auf die Neuschaffung oder den Wandel. Ab wann sich diese Vorstellung »historischer Garten« mit seiner bewahrenden Aufgabe als eine im soziologischen Sinne zu verstehende Institution durchsetzt, die nicht mehr hinterfragt (»taken-for-granted «) wird, ist keine ganz eindeutig zu beantwortende Frage. Wir werden darauf noch zurückkommen. Im nachfolgenden Zitat werden zuerst einmal die Anfänge der grundlegenden Bedeutungszuschreibung »historischer Garten « als Gartendenkmal, die diskutierten Alternativen anderweitiger Nutzung und das Ringen darum aufgezeigt:

Potsdam war auch deshalb so wichtig, weil die Gartendenkmalpflege als neue fachliche Aufgabe und »der historische Garten an sich" als neue Wertkategorie in der Diskussion nach 1918 in Potsdam entstanden sind, nämlich in der Diskussion nach dem Ende der Monarchie, was macht man mit den Residenzen und was macht man mit den Gärten. Das war 1924 ein großes Thema für den »Tag für Denkmalpflege« in Potsdam. »Was soll mit den Fürstenresidenzen passieren?« Es gab mehrere Tendenzen. Eine Position war: »Wir holen die Kunstsammlungen raus, tun sie in die Museen. Die Schlösser werden dann neuen Zwecken zugeführt als Hochschule, als Klinik, als was auch immer. Und die Gärten werden parzelliert und bebaut, und es wird jetzt also dem Volkswohl zugeführt." Das war eine Haltung, die sehr stark übrigens von den Museumskollegen verfolgt wurde, so im Sinne von: »Wir machen ein bisschen so was wie Leichenfledderei.«

Und dann gab es daneben die Denkmalpfleger, die gesagt haben, nein, das sind Gesamtkunstwerke, bestehend eben aus Haus und Ausstattung, und die müssen als organische Sachzusammenhänge oder Gesamtzusammenhänge auch erhalten bleiben. Das ist im Grunde genommen das Besondere. Es ist jetzt eben nicht das Schlossmuseum im Sinne von: "Das ist ein altes Schloss, da kann ich irgendwas reinhängen, alte Bilder, alte Möbel, irgendwas. «Das ist das Museumsschloss. Das war eine neue Typologie, die da entwickelt worden ist, ein neuer Begriff, dass nämlich das Schloss selber das Museum ist, also das Schloss als Großobjekt mit all seinen Ausprägungen im Inneren, dem historischen Mobiliar, den historischen Ausstattungsstücken und, und, und. Und in Potsdam ist dann zum ersten Mal auch erkannt worden, dass die Gärten dazugehören, und dass der Garten auch eine Denkmalkategorie ist. Denn bis dahin war das einfach Eigentum der herrschenden Familien, und die haben die Gärten genauso adaptiert für ihre Zwecke wie die Gebäude und haben natürlich nicht den Denkmalschutz gefragt. Für Potsdam gibt es zum Beispiel die schöne Geschichte, dass die Kaiserin die Sozialdemokratie verachtet hat und deshalb keine roten Blumen in Sanssouci wollte. Dort beginnt dann im Grunde genommen mit Georg Potente, dem Chef der Preußischen Gärten [Gartendirektor Parkrevier Sanssouci, ehemaliger Hofgärtner unter Wilhelm II.] in den 1920er Jahren, die historische Forschung. Und dann hat man auch angefangen, Gärten wieder zu rekonstruieren (weiterführend Dorgerloh 2004). 


\section{Phase b) \\ Die Kontinuitäten der gärtnerischen Fachpflege}

Historische Gärten sind auf beständige Pflege angewiesen. Das Bewahren eines bestimmten Zustandes in einem lebendigen Denkmal wie einem Garten ist trotz Revolutionen, Kriegen und Systemumbrüchen über viele Jahrzehnte hinweg maßgeblich auf Grund der hohen Kontinuität der Pflege durch die Gärtnerinnen und Gärtner gelungen. Die nach 1918 einmal angestoßene Etablierung einer Bedeutung als »historischer Garten « hätte ohne die kontinuierlich angewandten gärtnerischen Praktiken nicht fortbestehen können. Die Gärtner mit ihren Praktiken, mit ihrer Motivation für die Gartenpflege auch in Zeiten unsicherer Bezahlung sowie mit ihrer Wissensweitergabe über Generationen hinweg können als Repräsentanten der Institution »historische Gärten« betrachtet werden. Wir haben es hier also mit einer Institution zu tun, die sich sowohl in einem Raum, dem geographischen Ort des historischen Gartens, als auch in damit untrennbar verbundenen Praktiken der Gärtner mit ihren Dynastien an Gärtnerfamilien und einer notwendigen Bedeutungszuschreibung als Denkmal repräsentiert.

Die Gartenreviere gehörten zu den wenigen Teilen der Hofverwaltung, die auch nach dem Ende des Hofes weiter existierten. Als 1918 das Hauspersonal quasi entlassen wurde, blieb das Gartenpersonal irgendwie da. Viele wohnten auch da und haben sich halt weiter um diese Gärten gekümmert. Egal ob Revolution war oder nicht, die Orangerien wurden weiter beheizt und die exotischen Pflanzen gepflegt. Das Gleiche gab es noch mal 1945, als zum Ende des Zweiten Weltkriegs viele Mitarbeiter in den Gärten wohnen blieben und trotz Systemwechsel weiter ihre Arbeit erledigt haben, auch wenn gar nicht klar war, wer sie eigentlich bezahlt, nach dem Motto: »Die Hecke muss geschnitten werden, ob ich jetzt bezahlt werde oder nicht, es ist ja eine historische Hecke und ich kümmere mich darum. « Und das hat es übrigens 1989/90 noch einmal gegeben. Es gab also eine große personelle Kontinuität vor Ort. Zum Teil schon im 19. Jahrhundert begann die Entwicklung von Dynastien, die im Hofgärtnermuseum in Glienicke aufgezeigt werden. Die großen Gärtnerfamilien, etwa die Lennés, die Fintelmanns oder die Sellos, heirateten untereinander, wohnten vor Ort und bildeten sich gegenseitig aus. Es gab große Brüche, weil natürlich nicht mehr für den Hof gekocht wurde, aber es wurde sich weiter um die Anlagen gekümmert. Diese Kontinuität führte dazu, dass die Gärtner, die sich bisher auch um Neuanlagen gekümmert haben, jetzt das erhalten, was schon existiert. Sie pflegen das. Und das ist auch ein starkes Moment gewesen für die Bewahrung dieser Gartenanlagen, viel stärker als bei den Gebäuden.

Und nach 1945 hat sich das erstmal fortgesetzt. Es hat eine hohe Kontinuität gegeben, die sich aber eben in der DDR-Zeit aufgrund der ökonomischen Misere immer mehr auf bestimmte Flächen konzentrierte. Die Linie der Gartendenkmalpfleger war eben auch ganz klar: Wir müssen uns konzentrieren, wir machen bestimmte Sachen richtig. Und das war vor allen Dingen eben Sanssouci. Damit verbunden waren auch erste spektakuläre Rekonstruktionen in den 1970er und vor allem 80er Jahren. In Sanssouci betraf das vor allem die Schlossterrassen, wo die Veränderungen des 19. Jahrhunderts in eine Situation des 18. Jahrhunderts zurückgeführt wurden. Damit wurde etwas fortgeführt, was bereits nach 1918 angefangen hat, nämlich mit Blick auf den barocken Garten die späteren Veränderungen zurückzunehmen. Und da hat sich auch eine Kontinuität in der Gartendenkmalpflege gezeigt, die bis heute anhält. Aktuell ist in Sanssouci der östliche Lustgarten wiederhergestellt worden und das ist quasi die Fortsetzung einer denkmalpflegerischen Grundhaltung, die in den 1920er und 30er Jahren entwickelt worden ist. In dieser Tradi- 
tion arbeitet man bis heute. Also nicht nur personell, sondern auch fachlich, konzeptionell gibt es ganz lange Bögen, die sich ziehen lassen. Was die Einführung von anderen Fragestellungen oder auch das Einführen von anderen Methoden natürlich bei solchen langen Kontinuitäten durchaus erschwert.

\section{Phase c) \\ Die DDR und die Gartendenkmalpflege}

Die Vorstellung, dass diese Gärten Orte des historischen Bewahrens sind, verfestigt sich zu DDR-Zeiten weiter. Trotz Mangelwirtschaft werden Ressourcen mobilisiert, um vor allem das zentrale Gartendenkmal Sanssouci, aber auch in weiten Teilen den Neuen Garten zu bewahren. Pflegenotstände werden primär nicht als Missachtung des Wertes eines historischen Gartens an und für sich, sondern als Ergebnis der Mangelwirtschaft oder einer ohnehin übergeordneten staatlichen Grenzsicherung erlebt.

Bei meinen Ausführungen zur DDR und den historischen Gärten beziehe ich mich im Wesentlichen mit meiner Erfahrung auf Potsdam und die Gärten der Preußischen Residenzen, also Sanssouci, Neuer Garten, Babelsberg. Das sind meine Referenzobjekte, die ich auch zu DDRZeiten am stärksten wahrgenommen habe. Die DDR war eine Mangelwirtschaft. Und die Folge des Mangels sind schlechte Zustände. Diese gab es in der Bausubstanz, aber auch bei den Gärten. Aber die Gärten, die dann besonders gut gepflegt waren, und davon gab es wenige in der DDR, die wurden dann auch besonders wertgeschätzt. Sanssouci war zum Beispiel immer einer der am besten gepflegten Gärten in der DDR, und deshalb war es etwas Besonderes, dorthin zu gehen. Das Gleiche galt auch für den Neuen Garten in Potsdam, zumindest in Teilen. Für Park Babelsberg galt das nicht so, denn er lag ja im Schatten der Mauer. Babelsberg war auch nicht mehr erkennbar ein Schlosspark, sondern eher ein heruntergekommener Landschaftspark. Das war auch ein Ergebnis der Mangelwirtschaft, weil die Kollegen in der Potsdamer Schlösser- und Gärtenverwaltung gesagt haben: »Mit dem Wenigen müssen wir uns so konzentrieren, dass der bedeutendste Garten, nämlich Sanssouci, gut in Schuss ist, dann der Neue Garten noch; und Babelsberg, darum können wir uns nicht mehr kümmern, weil wir weder die personellen noch die ökonomischen Ressourcen haben, um die Anlagen in einem guten Zustand zu halten.«In der DDR konnte man ja auch nicht einfach so eine Firma beauftragen, es gab kaum Gartenbaufirmen. Es gab städtische Grünflächenämter, aber es gab damals eigentlich nur in wenigen Ausnahmefällen staatliche Gartenverwaltungen wie in Potsdam bzw. Sanssouci. Die Gärtner wurden aber anderweitig unterstützt, etwa durch freiwillige Arbeitseinsätze von Mitarbeitern oder Feierabendbrigaden.

Die DDR entwickelt sich zu einer international herausragenden Nation im Bereich der Gartendenkmalpflege. Die Zielvorstellung von aufwendigen historischen Rekonstruktionen verbreitet sich in den 1970er Jahren.

Die DDR hat in den 1970er Jahren angefangen, sich als eines der führenden Länder für die Gartendenkmalpflege zu etablieren. Historische Gartenforschung und auch Gartendenkmalpflege waren in der DDR im Verhältnis zur Fläche des Landes außerordentlich vorbildlich etabliert und es wurde auch sehr gut ausgebildet. Ich habe zum Beispiel an der Humboldt-Universität zu Berlin von einem Gartendenkmalpfleger Vorlesungen zur Geschichte der Gartenkunst gehört. Das gab es im Westen kaum. [...] Dazu kam, dass es in allen fünf Arbeitsstellen der Denkmalpflege in der DDR auch immer einen Bereich für die Restaurierungen und auch Erforschungen 
der historischen Gartenanlagen gab. Das war ungewöhnlich. Die westdeutschen Landesdenkmalämter hatten so etwas nicht. Und es gab in der Zentrale des Instituts für Denkmalpflege in Berlin auch eine eigene Abteilung für Gartengeschichte und Gartendenkmalpflege. Die Ausbildung dafür hat in Dresden an der Technischen Universität stattgefunden. Das Verständnis für die Wertigkeit der Gartenanlagen und auch der Gartendenkmalpflege war zu DDR-Zeiten nicht nur in Fachkreisen geradezu vorbildlich ausgeprägt.

\section{Phase d) \\ Die Wende, der Park Babelsberg und die Mauer}

Der Park Babelsberg war durch seine geographische Lage mitten im Grenzgebiet durch die Mauer zerschnitten. Hinzu kam die Idee, den Park zu einem Freizeitpark umzugestalten. Wir erkennen hier Tendenzen, die auch bereits nach 1918 diskutiert wurden: die Rückführung der ehemals herrschaftlichen Anlagen in eine dem aktuellen Zeitgeist entsprechende Nutzung als Volkspark. Die Bedeutung des Gartens als historisches Denkmal war offensichtlich auch zu DDR-Zeiten nicht unhinterfragt. Vielmehr können wir an diesem Beispiel den Deutungskampf zwischen dem Garten mit seiner Bedeutung als »historischer Garten « versus seine Bedeutung als »Volkspark« beobachten.

In Babelsberg war die Situation insofern anders, weil dort eigentlich schrittweise seit den 1950er Jahren die gesamte Parkanlage entwertet worden war. Die Akademie für Staats- und Rechtswissenschaften zog ein, im Schloss war die Filmhochschule untergebracht und es wurde als Studentenwohnheim genutzt und Neubauten wurden auch noch gebaut. Der Schlosspark sollte nach DDR-Muster ein Volkspark werden, mit Schwimmbad, Segelstation, Rodelbahn, Reitclub usw.

Dass dennoch die Referenz »historischer Garten« im Gegensatz zu »Volkspark « im Sinne der zuvor herausgestellten Linie der Kontinuität weiterhin vorhanden war, zeigen die bereits in der DDR von Enthusiasten begonnenen Aktivitäten der Wiederherstellung von Park Babelsberg als historischer Garten. Die anfänglich herausgearbeitete hohe Kontinuität der Bedeutungszuschreibung des Bewahrens scheint sich nicht als generalisierbare Überzeugung, nicht als unhinterfragte Institution in der DDR durchgesetzt zu haben. Es war aber für bestimmte Gruppen, allen voran die Gärtner und Denkmalpfleger, die unhinterfragbare Zielstellung. Auf Initiative Einzelner wurde nicht nur die praktische Arbeit zur Wiederherstellung des historischen Gartens geleistet; zugleich wurde damit eine Bedeutungsverschiebung im Sinne institutioneller Arbeit weg vom Volkspark, hin zum Gartendenkmal versucht. Befördert wurde die staatliche Gewährung dieser Aktivitäten für den Garten und die weitere Verfolgung der Bedeutungszuschreibung als historischer Garten durch die Möglichkeit, mit dem Thema Gartendenkmalpflege ein in hohem Maße unpolitisches Thema befördern zu können.

In den 1980er Jahren beginnt dann in Babelsberg die Wiederherstellung des historischen Gartens mit einer spektakulären Aktion im Pleasureground. Die rekonstruierten Wege liefen direkt auf den Grenzzaun zu und endeten dort unvermittelt, sodass alle gemerkt haben: »Da müsste es eigentlich weitergehen«; da gibt es auch tolles Fotomaterial dazu. Man hat dann kurzerhand 
mitten auf den Weg eine Bank gestellt, damit es nicht ganz so offenkundig ist. Es musste eigentlich ein Verrückter sein, der sagt: »Ich übernehme den Garten, und ich kümmere mich darum «; das war Karl Eisbein. Er hat mit kleineren Aktionen angefangen und eher so ein bisschen Robin-Hood- oder Greenpeace-mäßig, um den historischen Garten wieder ins Bewusstsein zu bringen. Als Schloss- und Parkführer wurden wir in den 1970er und 80er Jahren sehr stark in der Geschichte der Gärten ausgebildet. Wir sollten das ikonographische Programm von Skulpturen, die Gestaltungsprinzipien der Anlagen, die historischen Bezüge, die Pflanzenverwendung vermitteln, aber es ging überhaupt nicht um Preußen oder die Monarchie, und es sollte betont werden, dass diese Parkanlagen nicht nur für den Hof, sondern auch in hohem Maße öffentlich zugänglich waren. Das war die Art, wie man in der DDR, spätestens seit den 1960er, 70er Jahren, damit umging.

Interessant bleibt bei der Beschäftigung mit dem Deutungskampf zwischen »historischer Garten« und »Volkspark«, dass in bestimmten Kernzonen (Sanssouci, Neuer Garten) der Respekt vor dem historischen Garten sehr groß war. Das gute Benehmen im Garten war eine gelebte und dominierende Verhaltensweise. Fraglich ist, ob es wirklich der Respekt vor dem Garten als einem historischen Denkmal war, der zu DDR-Zeiten zu diesem Verhalten in diesen Parks geführt hat. Obgleich wir retrospektiv nur mutmaßen können, scheint es eine Mischung gewesen zu sein aus dem Respekt vor den Regeln des Obrigkeitsstaates und dem Respekt vor dem Gartendenkmal als Wert an und für sich. Die Differenzierung fällt schwer, weil beide Verhaltensmotivationen in die gleiche Richtung wirken, nämlich die Parkordnung zu respektieren. ${ }^{3}$ Zudem ist für Institutionen charakteristisch, dass einmal ausgeprägte und wiederholte Verhaltensweisen nicht mehr hinterfragt werden. Wo man sich schon immer beim Sonntagsspaziergang gut benommen hat, macht man es aus Gewohnheit weiter. Und wo schon immer im Winter gerodelt wurde, wird auch das beim nächsten Schneefall weiter tradiert.

\begin{abstract}
Also, es gab Sanssouci, und es gab den Neuen Garten mit einer Parkordnung und es gab die Parkwächter, die aufgepasst haben. Sie haben auch Bußgeld kassiert, und zwar fünf Mark, was viel Geld war in der DDR. Neben der Mangelwirtschaft war die DDR auch ein Obrigkeitsstaat, und da hat man sich halt an das gehalten, was die Obrigkeit vorgegeben hat. Danach war zum Beispiel Radfahren verboten, und man ging auch nicht auf die Wiesen. Und das war auch sehr eingeübt. Es war quasi die gute Stube, die nur zu bestimmten Gelegenheiten benutzt wurde. Am Sonntag ging man in der Sonntagskleidung in den Park oder machte einen Ausflug als Kollektiv oder mit der Familie und benahm sich auch entsprechend. Das heißt, es war nicht nur Teil des individuellen Freizeitverhaltens. Im Neuen Garten kann man das schön sehen. Da ist ja dann die Mauer reingebaut worden, also die ganze Grenzanlage. In diesem Bereich gab es eine kleine Stelle, wo man baden konnte. Das war aber nicht Teil des historischen Parks, sondern ein quasi verlorener Park, teilweise im unmittelbaren Schatten des Grenzzauns. Ansonsten ist keiner auf die Idee gekommen, direkt am Marmorpalais ins Wasser zu gehen. Das machte man einfach nicht. Man ging auch nicht bei Rot über die Straße. Das war eben antrainiert, quasi sozial kontrolliert. Und es gab natürlich auch Leute, die das sicherlich mal anders gehandhabt haben. Aber eben in den gut gepflegten Kernzonen dieser beiden Anlagen war das dezidiert nicht der Fall, dass man sich da von der Parkordnung abwegig verhalten hätte. In den Randbereichen, die zum Teil ziemlich ruinös waren, das Belvedere auf dem Pfingstberg oder der
\end{abstract}


Ruinenberg, also Bereiche, die erkennbar nicht gepflegt waren, da gab es natürlich Trampelpfade und da hat man alles Mögliche gemacht und sich auch irgendwie unbotmäßig benommen, im Sinne von auf Ruinen geklettert, rumgespielt, gerodelt und sonst was. Aber das war erkennbar kein Teil vom historischen Park, sondern man wusste: Das hat mal historisch dazugehört, war aber offensichtlich aufgegeben worden, es war außerhalb dieser gepflegten Kernzone. Sodass es für sportliche Aktivitäten, Freizeitaktivitäten, Indianerspielen und ich weiß nicht was genutzt wurde. Ich weiß gar nicht, ob die Parkordnung da offiziell auch gegolten hat, aber da hat man sich nicht dran gehalten. Und das macht eben auch einen Teil des Problems im Nutzerverhalten heute aus, dass eben diese Flächen in den Jahren nach 1990 dann sukzessive restauriert wurden. Damit ist man quasi in einen Bereich eingedrungen, der historisch zwar dazugehört hat, aber zwischenzeitlich aufgrund der ökonomischen Situation aufgegeben war. Und der deshalb eben auch durch eine andere Art von Nutzung erobert worden ist. Also, im Neuen Garten habe ich ganz viel gerodelt als Kind, obwohl der einigermaßen gut gepflegt war, und auch Schlittschuhlaufen ging man da. Und es ist natürlich total schwierig, dann auf einmal zu sagen: »Übrigens, Weltkulturerbe seit 1990, da wird nicht mehr gerodelt«, weil halt die Kinder von damals sind die Eltern von heute, und die gehen natürlich mit ihren Kindern dahin, wo sie schon immer gerodelt haben, ist ja logisch, wenn man in derselben Stadt wohnt. Das ist eines der Themen, wo man sagt: Also, die Probleme von heute hängen damit zusammen, dass es eben auch ein Flächenthema ist. Hätte die DDR die ökonomische und personelle Situation gehabt, dass sie größere Flächen hätte pflegen können, würde es vielleicht heute nicht diese Probleme geben, weil es damals auch schon quasi Tabuzone gewesen wäre.

Die Wende war mit großen Hoffnungen auf bessere Zeiten für die historischen Gärten verbunden. Die Vorreiterrolle der DDR in der Gartendenkmalpflege, die besondere Potsdamer Kulturlandschaft und die symbolische Aufladung als Verbindung zwischen Ost und West beförderten den erfolgreichen Welterbeantrag. Die Bedeutungszuschreibung als historisches Denkmal drängte vorerst die Ideen eines Volksparks weiter zurück.
Die Wende wurde für die historischen Gärten, jedenfalls für die Potsdamer Gärten kann ich es sagen, als eine ganz große Chance begriffen. Bei den staatlichen Schlössern und Gärten war eigentlich klar: "Jetzt geht es richtig los." Das ist in Potsdam durch die Aufnahme in die Welt- erbeliste 1990 sehr befördert worden. Der Antrag ist von der DDR für Potsdam, also für Sans- souci, Neuen Garten und Babelsberg gestellt worden. Mit der positiven Evaluation, da war dann schon die Mauer gefallen, sollte geprüft werden, ob nicht auch noch der Westteil dazukommen kann. Da hat der Berliner Senat in einer eher spontanen Aktion gesagt: »Wunderbar, wir treten dem Ostantrag bei«, und dann ist eben Glienicke und die Pfaueninsel mit dazugekommen.

Wie stark sich die Überzeugung durchsetzen konnte, dass die Gärten unhinterfragte Orte der Bewahrung einer vergangenen Zeitepoche sein sollten, wird am Park Babelsberg direkt nach der Wendezeit deutlich. Die Mauer wurde zurückgebaut und der ursprüngliche Gartenentwurf rekonstruiert. Zarte Versuche einer Diskussion, den Garten auch als Ort des Erinnerns an die Zeiten der innerdeutschen Grenze zu nutzen, verhallten ungehört. In diesem Moment mit seiner ganz spezifischen emotionalen Aufladung der Wendezeit wurde die Bedeutungszuschreibung »historischer Garten « nicht diskutiert. Wir werden an späteren Entwicklungen sehen, dass wir diese Unhinterfragbarkeit auf bestimmte Personenkreise, zuvorderst Gärtner und Denkmalpflegende, eingrenzen müssen.

Ja, das war also wirklich die Euphorie der Wendezeit. Da war es auch ganz klar, dass die Mauer abgerissen wird, mit allem, was dazugehört, und es war auch ganz klar, dass der historische 
Garten in diesem Bereich wiederhergestellt wird. Und es wurde nicht hinterfragt: »Ist das vielleicht eine wichtige geschichtliche Spur? « Nein, es gab nur eine Denkrichtung. Erstmals waren die Bereiche der Mauer wieder begehbar. Man konnte wieder von A nach B laufen oder im Sommer zwischen Berlin und Potsdam Boot fahren. Und dann war klar: Abbruch. Wiederherstellung, Modellierung. Und das war das, was der damalige Gartenchef Michael Seiler gemacht hat. Das Vorgehen wurde großzügig mit Fördermitteln unterstützt. Und es ist überhaupt nicht diskutiert worden. Es ging einzig um die Wiederherstellung von Sichtachsen, das Schließen von Wunden, das Wiedervereinen einer Kulturlandschaft; da gab es politisch Elogen rauf und runter. Eine große Rolle in der damaligen Diskussion spielte auch die Frage: Werden Berlin und Brandenburg ein gemeinsames Bundesland? Ja, so wie die Schlösser und Gärten zusammenwachsen, müssen auch die Institutionen zusammenfinden; und das war eigentlich auch die Blaupause für die Zusammenführung der beiden Schlösserverwaltungen und die Gründung der SPSG 1995. Und die Gärten waren da immer vorneweg. Bei denen war am schnellsten zu sehen, was für Erfolge zu erzielen waren. Es gab eine ganz große Begeisterung und überhaupt keine kritischen Stimmen im Sinne von »Ja, müsste man eventuell doch überlegen ob man alles rekonstruieren will ... und schade, dass die Mauer als schreckliches Zeugnis und als Ort des Erinnerns wegkommt. « Nein, die konnte gar nicht so schnell wie irgendwie nur abgeräumt werden. ${ }^{4}$

\section{Phase e) \\ Die Nachwendezeit und die Debatte um den Parkeintritt in Sanssouci}

Nach der Wende zeichneten sich Entwicklungslinien ab, die den konträren Bedeutungszuschreibungen mehr Raum gaben und durch Konflikte sichtbar machten. Nach dem Verschwinden der DDR-Obrigkeit wurde deutlich, dass die Regelbefolgung in den Kernzonen der historischen Gärten zum Teil auf eben sie zurückzuführen war und nur teilweise auf den Respekt vor dem Wert des zu bewahrenden Denkmals an und für sich. Die Regelbefolgung in Zeiten des Obrigkeitsstaats und die Bedeutungszuschreibung des Bewahrens haben sich zu DDR-Zeiten in die gleiche Richtung verstärkt und führten zur Befolgung der Parkordnung. Mit dem Ende der DDR fiel nicht nur der Obrigkeitsstaat weg, allem Obrigkeitsgebaren wurde fortan auch offen kritisch begegnet. Diese offene Kritik an Regelwerken ließ sich mit der Bedeutungszuschreibung als Volkspark mit seinen deutlich freieren Verhaltensweisen gut verbinden. Der nun offen ausgetragene Konflikt zwischen den konträren Bedeutungszuschreibungen des Volksparks einerseits und des Bewahrens andererseits wurde noch zugespitzt, weil die vormals zu DDR-Zeiten in den Randbereichen nicht gepflegten Gartenareale im Zuge des Welterbestatus wieder ein parkordnungskonformes Verhalten forderten. Die Bedeutungszuschreibung des Bewahrens erhebt also auch noch Anspruch auf die Areale mit jahrzehntelang tradierter Bedeutungszuschreibung als Volkspark.

Zum Thema des Verhaltens: Es gab nach der Wende eine große, sehr dankbare Inbesitznahme dieser wiedergewonnenen Landschaften als Wochenend- und Ausflugsgebiet, und zwar sowohl durch die West- und Ost-Berliner als auch die Potsdamer. Die Ossis hatten ja eben dieses Gefühl: Jetzt auf einmal ist die Freiheit da. Und dann hat sich das Freizeitverhalten massiv verändert. 
Die Gartenanlagen in den Mauerbereichen waren im Wesentlichen zehn Jahre nach dem Mauerfall wiederhergestellt. Dazu kam auch die Wiederherstellung der Bereiche, die man zu DDRZeiten aus ökonomischen Gründen nicht pflegen konnte, zum Beispiel der Ruinenberg. Und da kommt es dann natürlich zu Konflikten mit einer wachsenden Stadt, einer Stadt mit Bevölkerungsdruck, einer Stadt, in der sich Gewohnheiten ausgebildet haben. Da kommt es zu Spannungen, wenn etwa der traditionelle Berg zum Schlittenfahren auf einmal nicht mehr Rodelberg, sondern Teil des Welterbes ist. Als mein Vorgänger versucht hat, die Parkordnung restriktiv durchzusetzen, ist es in den späten 1990er Jahren zu massiven Konflikten gekommen, im Sinne von »Das ist unser Steuergeld, mit dem das gemacht wird; das ist unser Park, und wir wollen den nutzen; und wir wollen den auch so nutzen, wie wir das wollen. "Und da setzte im Grunde die Debatte über die Frage ein, wie wir mit den historischen Gärten umgehen wollen.

Die Diskussion um den Umgang mit den historischen Gärten entfachte sich für Sanssouci vor allem an der Forderung nach einem Parkeintritt, der einen klaren Unterschied zu einem Volkspark signalisiert. Ein Eintritt zeigt zum einen an, dass etwas Wertvolles geschützt werden soll oder mit gebührender Distanz besichtigt werden darf. Zum anderen wird mit dem Betreten des Areals ein entsprechendes Verhalten eingefordert; in Analogie zum Flüstern im Museum und zum bedächtigen Durchschreiten einer Kunstausstellung. Mit dem Parkeintritt für Sanssouci sollte die Pflege finanziert werden. Die Forderung, ihn zu erheben, kann als ein Versuch interpretiert werden, die Bedeutungszuschreibung des Bewahrens zu stärken und diejenige eines Volksparks zurückzudrängen. Der Deutungskampf bricht mit dieser Debatte an die Oberfläche. Obgleich sich die Forderung nach einem Parkeintritt nicht durchsetzen konnte, wurde das Bewusstsein für den Wert des Bewahrens auf einer diskursiven Ebene gestärkt, gleichwohl die Regelbefolgung sich nur marginal erhöht hat und die Ebene der Alltagspraktiken davon unberührt blieb. Die Diskursverschiebung hat unter anderem den Boden bereitet für weitere finanzielle Zuwendungen.

2004 habe ich [als Generaldirektor der SPSG] mit der Forderung nach einem Parkeintritt noch eins draufgesetzt: »Übrigens, wir haben jetzt viel mehr Fläche, die wir pflegen müssen«; denn es gab immer Geld für die Investitionen und kein Geld für die Pflege. Wir haben also ein massives Pflegedefizit in Potsdam, ja, bis heute eigentlich; und dann war die Frage: »Wenn wir das irgendwie pflegen wollen, müssen wir es anders finanzieren«, und es gab 2002 oder 2003 natürlich überhaupt keine zusätzlichen Stellen. Also lag nahe: »Wir nehmen Eintritt, und aus dem Eintritt wird die Parkpflege finanziert.« Das ging nun aber natürlich konträr gegen dieses: »Wir haben endlich diese Räume wieder, und jetzt wollt ihr, dass wir da Eintritt zahlen.« Das ging irgendwie gar nicht zusammen. Aus heutiger Sicht halte ich das auch für völlig nachvollziehbar. Damals war ich der Meinung: »Wieso, das ist doch ganz toll, und ihr wolltet das alle; das Wiedergewinnen des Historischen, auch in einer bestimmten Qualität, und das muss doch auch gepflegt werden, und dann, bitteschön, müssen wir doch alle dazu einen Beitrag leisten.« Und dass ich dann gesagt habe: »Ihr könnt jetzt auf ein paar Strecken Fahrrad fahren«, war überhaupt nicht zu kommunizieren, im Sinne von: »Wir kommen euch entgegen, dass ihr auf ein paar Strecken Fahrrad fahren könnt.« Sondern es wurde aufgefasst nach dem Motto: »Wieso? Wir fahren doch jetzt hier seit Jahren überall Fahrrad.« Es gab keine Parkwache mehr, es gab auch keine Restriktionen mehr. »Wieso grenzt der uns denn jetzt schon wieder ein, und wir dürfen nicht mehr überall Fahrrad fahren? « Es war kommunikativ nicht möglich zu sagen: »Wir kommen euch ganz toll entgegen. "Sondern: "Wieso? Wir fahren doch jetzt hier schon. "So: "Wie, wo, was? Das ist doch alles alter Osten, Obrigkeitsstaat, Diktatur, da haben die uns schon lange genug vorgeschrieben, was wir eigentlich machen wollen. Jetzt, hier: freier Park für freie Bür- 
ger.« Das ist schon eine ganz schwierige Situation gewesen und hat die Gärtner ziemlich demoralisiert, die das Gefühl hatten: »Keiner schätzt eigentlich unsere Arbeit. « Das hat also doch ein hohes Maß an Unzufriedenheit, Unverständnis und auch innerinstitutionell ganz massiven Druck in meine Richtung ausgelöst: »Wieso? Der Chef erlaubt jetzt irgendwie das Fahrradfahren? Ist der denn wahnsinnig? Der macht ja alles nur noch viel schlimmer! Warum setzt der sich denn jetzt nicht durch? Und das muss aber mal klar gesagt werden: Hier herrschen wieder Zucht und Ordnung. « Also, das war auch innerhalb der Institution ziemlich schwierig. Dass zum Beispiel Marketingleute dann gesagt haben: „Okay, wir erfinden jetzt mal so was wie die Potsdamer Schlössernacht. "Das war zum Beispiel eine Erfindung, die deutlich machen sollte: »Wir können diesen Park auch zu besonderen Ereignissen besonders nutzen. Wir stellen die Parkanlagen auch für Feste zur Verfügung; wir machen nicht zu; wir schließen nicht ab; wir öffnen uns auch. "Kostete dann aber eben 30-40 Euro oder Mark zunächst. Also, diese Schlössernächte waren ja, 2000 gab es die erste, ein riesiger Erfolg.

Am Parkeintritt hat sich ganz viel entzündet. Und es war aber auch eine gute Möglichkeit, über den historischen Garten zu berichten, und auf Pflegedefizite und Probleme aufmerksam zu machen. Es hat bloß nicht dazu geführt, dass Leute gesagt haben: »Ja super, dann gehe ich jetzt eben nicht mehr baden und mache nicht meine Grillparty und dann fahre ich Fahrrad nur noch auf den dafür zugelassenen Wegen.«

\section{Zu Phase f) \\ Das Thema Klimawandel und die historischen Gärten in Berlin-Potsdam}

Die Diskussion um den Parkeintritt ist anders gelagert als die gegenwärtig geführte Debatte um den Klimawandel. Wir kehren also am Ende des Beitrags zurück zu unserer Ausgangsfrage, inwieweit der Klimawandel zu einem expliziten Thema für die historischen Gärten in Berlin-Brandenburg bzw. Ostdeutschland geworden ist. In den bisher herausgearbeiteten Entwicklungslinien zweier konträrer Bedeutungszuschreibungen fügt sich das Thema Klimawandel befördernd und gerade nicht konflikthaft ein. Unabhängig davon, ob dem Garten die Bedeutung des Bewahrens oder die als Volkspark zugesprochen wird, ist der Klimawandel eine Bedrohung. Das vorhandene Grün ist auf bestimmte klimatische Bedingungen angewiesen, und in Zeiten von längeren Hitzeperioden wird der Wunsch nach Grün für alle dringlicher. Das Thema Klimawandel zeigt sich also als notwendigerweise zu bearbeitende Problemstellung, um den historischen Garten zu erhalten.

Das Thema Klimawandel fügt sich aber nicht einfach konfliktfrei in die bisherigen Bedeutungszuschreibungen ein. Das wäre zu vereinfachend. Damit einher geht durchaus ein Versuch, Wandel anzustoßen und eine Bedeutungserweiterung ergänzend zum Bewahren anzuregen. Es ist der Versuch, den historischen Garten als »Brennglas für gesellschaftsrelevante, aktuelle Themen « zu sehen. Obgleich sowohl der historische Garten als auch der Volkspark ein vom Klimawandel bedrohtes öffentliches Grün sind, sind die historischen Gärten stärker betroffen. So können beispielsweise historisch verwendete Gehölze von semantischer Bedeutung im Sinne des Bewahrens kaum durch robustere ersetzt werden. Deshalb verdeutlichen sich auch die allgemeinen Gefahren im Natur- und Umweltschutzbereich in besonderer Weise im historischen Garten. Die Debatte um den Klimawandel kann also auch 
zu einer Verschiebung in der Wahrnehmung von historischen Gärten beitragen: Gerade das in die Vergangenheit verweisende Bewahren macht besonders deutlich, welche aktuellen und zukünftigen Herausforderungen mit dem Klimawandel bewältigt werden müssen.

Zudem liefert das Thema Klimawandel im Verbund mit der Positionierung historischer Gärten als Brennglas gesellschaftsrelevanter Themen ein überlokal anwendbares Template für andere Gärten. Im Sinne einer Theoretisierung wird eine lokale Bedeutungserweiterung (Bewahren und gesellschaftlich relevant sein) abstrakt genug dargeboten, um auch für andere Gärten eine Veränderung der Bedeutungszuschreibung zu ermöglichen. Das damit einhergehende überregionale Interesse gibt weiteren Aufwind (Greenwood et al. 2002).

Das Thema historische Gärten und Klimawandel ist, glaube ich, ein gutes Beispiel, wie lange Wandel auch dauert ... wie langsam das auch gehen kann. Die Besetzung dieses Themas war ein Anliegen von mir, weil ich gesagt habe: Wenn wir die historischen Gärten rausholen wollen aus der Ecke »Im Garten, da mach ich, was ich will. Und das wächst ja sowieso alles wieder nach«, dann brauchen wir einen anderen Zugang zu dem Thema, und wir brauchen andere Verbündete, und das kann nicht der Naturschutz sein, weil der andere Ziele verfolgt, sondern wir brauchen nochmals ein anderes Netzwerk oder andere Stakeholder, die auch sagen: »Mensch, der hat ein interessantes Thema." Und was wir eben haben, sind diese Wetterextreme, biologische Invasion und so weiter, kombiniert mit diesem hohen Nutzungsdruck. Als wir 2012 wieder einen großen Kongress zu den Gärten planten, dachte ich: aber bitte nicht wieder zur Gartengeschichte, nicht wieder zur Gartendenkmalpflege, sondern: „Was ist ein aktuelles, gesellschaftlich relevantes Thema? Hat das mit diesem Klimawandel und diesen historischen Gärten eigentlich schon jemand untersucht?« Wir haben ein Phänomen, wir können das noch nicht genau beschreiben; wir haben keine Patentlösung, es wird auch keine geben; wir brauchen aber eine andere Art von öffentlicher Wahrnehmung des Problems; wir brauchen andere Partnerschaften; wir müssen sehen, wer sich dafür vielleicht noch interessiert. Und möglicherweise führt das zu Hilfestellungen für die praktischen Lösungen, vielleicht ist aber auch die Diskussion ja schon einfach mal hilfreich. Wir wollen im Grunde genommen ja nicht über die Symptome reden, sondern wir wollen eigentlich auch sagen, diese historischen Altlasten haben auch eine Bedeutung für die Gesellschaft heute und vielleicht auch morgen; und wenn wir die deshalb erhalten wollen, dann müssen wir uns überlegen, ob wir unser Verhalten ändern, nicht nur in den historischen Gärten, sondern überhaupt in der Welt, denn der Klimawandel ist ja nicht gottgegeben. Wir sind ja diejenigen, welche verantwortlich sind. Und wir sorgen einerseits dafür, dass es jetzt irgendwie diese Trockenperioden gibt, mal im übertragenen Sinne, und rennen dann auch noch hin und machen die Grasnarben kaputt. Und wir finden das dann aber ganz schrecklich, wie es danach aussieht. Das ist doch irgendwie ziemlich schizophren. Also, wenn man dort rausgeht und sagt: "Ich kaufe mir jetzt doch vielleicht drei T-Shirts weniger in der Saison «, dann wäre das ja schon ein wichtiger Erfolg. Das wäre auch ein wichtiger Erfolg für die Gärten, wenn man sagt: »Übrigens, je nachdem, wie ich mich hier verhalte, hat das im Endeffekt Auswirkungen auch vor meiner Haustür «, sei es die Erkenntnis, ja ... »Fast Fashion ist ein umweltbelastendes Unterfangen, oder auch meinen Elektronikschrott nach Nigeria zu schicken«. Und wenn man das ein Stück weit kontextualisiert ... Die biologische Invasion ist ja auch ein Indikator dafür, genauso wie die Wetterextreme, dass historische Gärten eigentlich nur ein Brennglas sein können, wo man die Auswirkungen dieser globalen Kontexte auf unser heutiges Dasein vielleicht mit einer größeren Eindringlichkeit transportieren kann. Es gibt ja nach wie vor diese unglaublich hohe Zustimmung zu Bedeutung und zum Wert, alle finden diese Gärten toll. Aber dass die eben in so einer Situation der Gefährdung sind, und zwar nicht aufgrund mangelnder Pflege oder weil wir nicht wissen, wie bedeutend die sind ... das sind eigentlich alles schon die Themen von gestern, sondern dass wir sie quasi in einer sehr gefährdeten, ja geradezu global 
gefährdeten Situation haben; das ist etwas, was man über diese Interdisziplinäre Arbeitsgruppe Historische Gärten im Klimawandel auch in bestimmte Felder bringt. Ein Effekt der Arbeitsgruppe liegt für mich darin, dass jetzt andere Naturwissenschaftler, Geoforscher, Sozialwissenschaftler auf einmal dieses Thema spannend finden. Das ist eigentlich für mich der größte Effekt. Ich erhoffe mir, dass das Thema eben nicht nur von den Gartenexperten diskutiert wird. Das ist im Grunde genommen der Ansatz dafür.

\section{Zusammenfassung und Ausblick}

In Bezug auf die uns beschäftigende Frage, inwieweit der Klimawandel zu einem expliziten und ernstzunehmenden Thema für die historischen Gärten in Berlin-Brandenburg bzw. Ostdeutschland wird, können wir im Kontext der bisherigen Entwicklungslinien einer Bedeutungszuschreibung historischer Gärten im Zeitverlauf eine positive Prognose ausstellen. Die bereits mit der Abschaffung der Monarchie entfachte Debatte um die Bedeutung der Gärten als Gartendenkmal oder Volkspark ist bis heute aktuell. Im Zeitverlauf können wir beobachten, wie die Arbeit an der Institution des historischen Gartens der Bedeutung als Gartendenkmal mal mehr und mal weniger Vorschub geleistet hat. Obgleich zu DDR-Zeiten die Gartendenkmalpflege hochrenommiert war und mit Sanssouci und dem Neuen Garten vorbildliche Gartendenkmalpflege bewiesen wurde, lief doch auch der Gedanke an die Etablierung eines Volksparks etwa für Babelsberg immer unterschwellig mit. Die Einzelinitiativen zur Rückeroberung des Parks Babelsberg als Gartendenkmal waren wichtige Schritte einer Bedeutungsverschiebung. Die Wendezeit konnte zwar in der Anfangseuphorie auch aufgrund der besonderen Örtlichkeit in Ost-West mit dem Rückbau der Mauer die Bedeutung des Bewahrens noch einmal stärken. In der Nachwendezeit wurde der Deutungskampf jedoch wieder stärker. Er kulminierte symbolisch in den Diskussionen um den Parkeintritt. Der Klimawandel ist als Thema ungeachtet des nach wie vor nicht entschiedenen Deutungskampfs relevant. Die damit einhergehende Bedeutungserweiterung, dass historische Gärten mehr sind als Gartendenkmale, sondern Brenngläser gesellschaftsrelevanter Problemlagen, eröffnet spannende Perspektiven. Es ist den Gärten zu wünschen, dass sie damit weiter in das Zentrum gesellschaftlicher Aufmerksamkeit rücken und ihnen die Wertschätzung entgegengebracht wird, die für ihre Zukunftssicherung notwendig ist. Es wird noch einige institutionelle Arbeit notwendig sein, um dieses Ziel zu erreichen.

\section{Literatuverzeichnis}

DiMaggio, Paul J. (1988): Interest and Agency in Institutional Theory. In: Institutional Patterns and Organizations: Culture and Environment. Hg. von Lynne G. Zucker. Cambridge, MA: Ballinger, 3-21.

Dorgerloh, Hartmut (2004): Hofgärten zu Volksgärten? Die königlichen Gärten nach dem Ende der Monarchie und die Anfänge der Gartendenkmalpflege von 1918 bis 1927. In: Wege zum Garten. Gewidmet Michael Seiler zum 65. Geburtstag. Hg. von der Generaldirektion der Stiftung Preußische Schlösser und Gärten Berlin-Brandenburg. Leipzig: Koehler \&Amelang, 255-261. 
Dorgerloh, Hartmut (2006): Warum ist die Mauer weg? Preußische Schlösser und Gärten und die Behandlung ihrer Zeitschichten. In: Brandenburgische Denkmalpflege 15.1. Berlin: Arenhövel, 35-38.

Dorgerloh, Hartmut (2016): Pücklers Erbe in Babelsberg - Bewahrung und Wiederherstellung im UNESCOWelterbe. In: Parkomanie. Die Gartenlandschaften des Fürsten Pückler in Muskau, Babelsberg und Branitz, Hg. von der Kunst- und Ausstellungshalle der Bundesrepublik Deutschland GmbH Bonn. München et al.: Prestel, 186-191.

Greenwood, Royston; Suddaby, Roy; Hinings, Christopher R. (2002): Theorizing change: the role of professional associations in the transformation of institutionalized fields. In: Academy of Management Journal 45.1, 58-80.

Greenwood, Royston; Oliver, Christine; Lawrence, Thomas B.; Meyer Renate E. (2017²) (Hg.): The Sage Handbook of Organizational Institutionalism. London, CA: Sage.

Hampel, Christian E.; Lawrence, Thomas B.; Tracey, Paul $\left(2017^{2}\right)$ : Institutional Work: Taking Stock and Making it Matter. In: Greenwood et al. (Hg.) $\left(2017^{2}\right), 558-590$.

Hoffman, Andrew J. (1999): Institutional Evolution in Change. Environmentalism and the U.S. Chemical Industry. In: Academy of Management Journal 42.4, 351-371.

Lawrence, Thomas B.; Suddaby, Roy $\left(2006^{2}\right)$ : Institutions and institutional work. In: Handbook of Organization Studies. Hg. von Stewart R. Clegg, Cynthia Hardy, Thomas B. Lawrence und Walter R. Nord. London: Sage.

Maguire, Steve; Hardy, Cynthia (2009): Discourse and deinstitutionalization: The decline of DDT. In: Academy of Management Journal 52.1, 148-178.

Munir, Kamal A.; Phillips, Nelson (2005): The birth of the `Kodak moment $<$ Institutional entrepreneurship and his adoption of new technologies. In: Organization Studies 26.11, 1665-1687.

Zilber, Tammar B. (2017): The evolving role of meaning in theorizing institutions. In: Greenwood et al. (Hg.) $\left(2017^{2}\right), 418-445$. 
